Traditional Medicine and Modern Medicine

Vol. 1, No. 2 (2018) 137-143

(C) Institutes of Integrative Medicine, Fudan University and

World Century Publishing Corporation

DOI: $10.1142 /$ S2575900018500076

\title{
Development of clinical risk models for diabetic cardiovascular autonomic neuropathy in a Chinese population using logistic regression analysis
}

\author{
Lin Jiang ${ }^{1, \S}$, Genlong $\mathrm{Wu}^{2, \S}$, Ping Fang ${ }^{3}$, Zhensheng $\mathrm{Xu}^{1, *}$ and Zihui Tang ${ }^{3, \dagger}$, \\ ${ }^{1}$ Health Management Center, Huashan Hospital \\ Fudan University, Shanghai, P. R. China \\ ${ }^{2}$ Qingpu Hospital of Traditional Chinese Medicine, Shanghai, P. R. China \\ ${ }^{3}$ Department of Endocrinology and Metabolism \\ Shanghai Tongji Hospital, Tongji University School of Medicine \\ Shanghai, P. R. China \\ *xu2000xu@126.com \\ †albert.tang@163.com
}

Received 18 December 2017; Accepted 3 May 2018; Published 21 June 2018

\begin{abstract}
Background: We developed clinical risk models for predicting diabetic cardiovascular autonomic neuropathy (DCAN) in Chinese diabetic patients.

Methods: A Chinese cohort of 455 diabetic participants underwent a short heart rate variability (HRV) test which was recruited between 2011 and 2013. Clinical risk models were developed that included independent and significant risk factors by using multiple variable stepwise regressions. These clinical risk models were tested in another independent cohort of Chinese individuals.

Results: The clinical risk models included age, fasting plasma glucose, 2-h plasma blood glucose, triglycerides, resting HRs, and duration of diabetes mellitus. The area under the receiver-operating characteristic (ROC) curve of the study group was 0.794 . In the model with the continuous variables, the area under the ROC curve was 0.810 . A cutoff score of 12.54 which produced the optimal sensitivity $(68.20 \%)$ and specificity $(76.80 \%)$ and identified the percentage $(35.77 \%)$ of the population that required subsequent testing.
\end{abstract}

Conclusions: The clinical risk models showed high sensitivity and specificity for the prediction of DCAN in Chinese diabetic patients.

Keywords: Diabetes cardiovascular autonomic neuropathy; clinical risk model; Chinese diabetic patients; logistic regression analysis.

\section{Introduction}

Cardiovascular autonomic neuropathy (CAN) is considered a common diabetic chronic complication. ${ }^{1}$ Among patients with diabetes, the prevalence of CAN ranges from $2.6 \%$ to $90 \%$, depending on the methodology and diagnostic criteria applied. ${ }^{2}$ Diabetic CAN (DCAN), which results in damage to neural fibers responsible for innervation of the heart and cardiac vessels, can lead to atypical clinical manifestations. ${ }^{3}$ Due to the insidious onset of DCAN and the absence of typical symptoms, the diagnosis of this disease is often delayed, and the prevalence of the disease is underappreciated.

$\ddagger$ Corresponding authors.

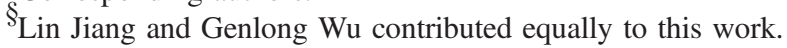


CAN significantly increases the risk of fatal and non-fatal cardiovascular events and is associated with increased mortality. ${ }^{4-6}$ The Action to Control Cardiovascular Risk in Diabetes (ACCORD) study showed that the mortality rate of patients with CAN was approximately 1.55-2.14 times higher than that of patients without CAN. ${ }^{6}$

An individualized approach to risk estimation of DCAN is needed to aid risk stratification and identify high-risk individuals who may benefit from intensive interventions. Over the past decades, many risk scores have been developed to predict cardiovascular disease ${ }^{7-9}$ and apply a prediction model in clinical practice. We previously developed clinical risk models for predicting CAN in a general Chinese population. ${ }^{10}$ Our findings indicated that glucose profiles, lipid profiles, and blood pressure (BP) profiles were significantly associated with DCAN, ${ }^{1-13}$ suggesting that these factors may predict the development of DCAN in diabetes patients. Additionally, a screen model with high sensitivity and specificity for DCAN had been developed. ${ }^{14}$ However, to the best of our knowledge, in Chinese diabetic patients, there have been no reports of clinical risk models to predict DCAN. Clinical risk models with high sensitivity and specificity are needed to predict DCAN and alert clinicians to the need for further tests. The purpose of this study was to develop and validate clinical risk models for DCAN in Chinese diabetic patients. An additional aim was to evaluate the performance of these clinical risk models for DCAN.

\section{Materials and Methods}

\section{Study population}

As is mentioned in our previous studies, ${ }^{11-14}$ a DCAN risk factor analysis in a Chinese random sample was carried out using established methods. Diabetic participants aged 30-80 years with undiagnosed CAN were recruited from rural and urban communities in Shanghai. More than 500 participants were invited to a screening visit between 2011 and 2013. To eliminate potential confounding factors that could influence cardiovascular autonomic (CA) function, some subjects were excluded from the study. The exclusion criteria were mentioned in previous studies ${ }^{11-14}$ : (1) a history or findings of arrhythmia and hyperthyroidism or hypothyroidism, (2) pregnancy or lactation, or (3) serious hepatic or renal dysfunction. Of these subjects, 455 diabetic participants with complete clinical baseline data were available for the risk analysis of DCAN. For validation of the clinical risk model of DCAN in an external dataset, 115 diabetic patients who fulfilled the same inclusion and exclusion criteria were recruited from health examination centers. Written consent was obtained from all the patients before the study, and this study was approved by the Ethics Committee of Huashan Hospital, Shanghai, China.

\section{Measurements}

As done in previous studies, ${ }^{11-13}$ the patients were interviewed to collect data on their medical histories and medication, smoking habits and results of laboratory assessments of cardiovascular disease risk factors. All the subjects underwent a complete evaluation after an 8-h fast. This included medical history and a physical examination, in addition to heart rate (HR), BP, fasting serum glucose, insulin, and fasting plasma lipid measurements. The patients' demographical information, results of laboratory assays, HR variability (HRV indices), and medical histories have been detailed earlier. ${ }^{1-13}$ In all the analyses, the day-to-day and inter-assay coefficients of variation at the central laboratory in our hospital were between $1 \%$ and $3 \%$. The definitions of hypertension (HTN), body mass index (BMI), diabetes mellitus (DM), and metabolic syndrome (MetS) were the same as those described elsewhere. ${ }^{15,16}$

In this large-scale population-based study, prior to a CA function assessment, the participants were advised to avoid alcohol, smoking, and coffee for $24 \mathrm{~h}$, so as not to influence their resting status. To evaluate CA function and changes in autonomic regulation, a short HRV test was applied using a computer-aided examination and spectral analysis system. DCAN was diagnosed based on at least two abnormal CA reflex test results. ${ }^{15,16}$

\section{Development and validation of the clinical risk models}

The methods used to develop the clinical risk models for DCAN were similar to those employed in our previous study. ${ }^{10}$ Briefly, the protocol for generating the clinical risk models was as follows: (1) collection of potential risk factors for DCAN; (2) estimation of significant risk factors using univariate regression analysis; (3) development of best-fit models via multiple variable regression analysis in an exploratory dataset; (4) development of the clinical risk models; and (5) validation of the clinical risk models in the study group and external cohort.

Potential clinical risk factors reported in the literature to be associated with DCAN were included in the analysis. Univariate analyses were performed to estimate significant predictors of DCAN. Multiple logistic regression (MLR) was used to compute $\beta$-coefficients for known risk factors for DCAN. The best-fit model of the selected risk factors was created using MLR with stepwise methods. Variables significant at $10 \%$ were included in the MLR using stepwise 
Table 1. The baseline of characteristics of participants.

\begin{tabular}{lccccc}
\hline Variable & Entire sample & Female & Male & $P$ value* & External sample \\
\hline$N$ & 455 & 247 & 208 & - & 115 \\
Age year & $62.8 \pm 8.61$ & $62.17 \pm 8.37$ & $63.54 \pm 8.84$ & 0.016 & $58.34 \pm 9.9$ \\
Height cm & $162.12 \pm 8.15$ & $157.2 \pm 5.99$ & $167.95 \pm 6.33$ & $<0.001$ & $163.31 \pm 8.84$ \\
Weight kg & $66.63 \pm 11.65$ & $62.9 \pm 11.3$ & $71.05 \pm 10.45$ & $<0.001$ & $65.35 \pm 12.59$ \\
SBP mmHg & $134.3 \pm 20.3$ & $134.95 \pm 21.33$ & $133.55 \pm 19.02$ & 0.305 & $131.88 \pm 20.1$ \\
DBP mmHg & $81.08 \pm 10.12$ & $81.2 \pm 10.1$ & $80.93 \pm 10.16$ & 0.69 & $76.72 \pm 11.08$ \\
FPG mmol/L & $7.34 \pm 2.69$ & $7.11 \pm 2.56$ & $7.61 \pm 2.82$ & 0.006 & $9.23 \pm 5.08$ \\
PBG mmol/L & $11.98 \pm 4.42$ & $11.9 \pm 4.25$ & $12.07 \pm 4.62$ & 0.583 & $13.07 \pm 5.44$ \\
FINS mIU/L & $10.45 \pm 24.39$ & $11.09 \pm 24.53$ & $9.68 \pm 24.23$ & 0.388 & $22.57 \pm 56.21$ \\
TC mmol/L & $5.38 \pm 1.11$ & $5.64 \pm 1.08$ & $5.06 \pm 1.07$ & $<0.001$ & $4.63 \pm 1.21$ \\
TG mmol/L & $1.99 \pm 1.18$ & $1.99 \pm 1.03$ & $1.99 \pm 1.34$ & 0.961 & $1.99 \pm 1.43$ \\
HDL mmol/L & $1.3 \pm 0.31$ & $1.38 \pm 0.29$ & $1.19 \pm 0.28$ & $<0.001$ & $1.23 \pm 0.53$ \\
LDL mmol/L & $3.28 \pm 0.85$ & $3.4 \pm 0.86$ & $3.14 \pm 0.82$ & $<0.001$ & $3 \pm 1.14$ \\
SCr $\mu$ mol/L & $81.37 \pm 24.04$ & $73.35 \pm 23.1$ & $90.93 \pm 21.54$ & $<0.001$ & $72.67 \pm 32$ \\
UA $\mu$ mol/L & $298.09 \pm 85.47$ & $280.17 \pm 77.45$ & $319.48 \pm 89.66$ & $<0.001$ & $309.46 \pm 100.85$ \\
HR bpm & $75.11 \pm 10.41$ & $74.96 \pm 9.63$ & $75.29 \pm 11.27$ & 0.634 & $73.26 \pm 11.41$ \\
TP ms ${ }^{2}$ & $747.3 \pm 682.53$ & $763.34 \pm 635.42$ & $728.25 \pm 734.89$ & 0.44 & $658.07 \pm 680.86$ \\
LF ms ${ }^{2}$ & $166.57 \pm 225.93$ & $152.57 \pm 145.95$ & $183.19 \pm 293.24$ & 0.042 & $145.83 \pm 225.52$ \\
HF ms ${ }^{2}$ & $152.15 \pm 188.51$ & $163.58 \pm 193.2$ & $138.57 \pm 182.1$ & 0.046 & $119.25 \pm 174.13$ \\
LFHF & $1.84 \pm 2.12$ & $1.66 \pm 1.91$ & $2.05 \pm 2.33$ & 0.006 & $1.98 \pm 2.36$ \\
Smoking & $4(1.62 \%)$ & $85(40.87 \%)$ & $89(19.56 \%)$ & $<0.001$ & $13(22.81 \%)$ \\
DMD year & $5.24 \pm 6.45$ & $4.86 \pm 6.29$ & $5.73 \pm 6.62$ & 0.063 & $10.57 \pm 8.23$ \\
HTN yes,\% & $159(64.37 \%)$ & $132(63.46 \%)$ & $291(63.96 \%)$ & 0.776 & $67(58.77 \%)$ \\
HTND year & $6.42 \pm 9.99$ & $5.62 \pm 9.05$ & $7.41 \pm 10.96$ & 0.008 & $6.12 \pm 8.72$ \\
MetS yes, $\%$ & $187(75.71 \%)$ & $143(68.75 \%)$ & $330(72.53 \%)$ & 0.019 & $87(75.65 \%)$ \\
DCAN yes, $\%$ & $67(27.13 \%)$ & $65(31.25 \%)$ & $132(29.01 \%)$ & 0.172 & $47(40.87 \%)$ \\
\hline
\end{tabular}

“*” presents the difference between female and male. SBP: systolic blood pressure, DBP: diastolic blood pressure, FPG: fasting plasma glucose, PBG: plasma blood glucose, FINS: fasting blood insulin, TC: serum total cholesterol, TG: triglyceride, HDL: high-density lipoprotein cholesterol, LDL: low density lipoprotein cholesterol, SCr: serum creatinine, HR: heart rate, TP: total power of variance, LF: low frequency, HF: high frequency, DMD: duration of diabetes mellitus, MetS: metabolic syndrome, HTN: Hypertension, DCAN: diabetic cardiovascular autonomic neuropathy, UA: uric acid.

backward elimination, with DCAN as the dependent variable. The independent variables were categorized. A $P$ value $\leq 0$. 10 was considered significant. A clinical risk model was based on a sum score derived from the addition of the score for each variable in the risk model for each participant. One clinical risk model including the independent factors with continuous variables (model 1) was developed, and another was developed including the independent factors with categorical variables (model 2).

As mentioned in our previous studies, ${ }^{10,17-19}$ the performance of the clinical risk models was evaluated in the study group and external cohort. A receiver-operating characteristic (ROC) curve and area under the curve (AUC) were produced. The sensitivity and specificity were calculated for each cutoff score. The cutoff score that gave the maximum sum of sensitivity and specificity (Youden index) was taken as the optimum. In addition, the cutoff scores for a low and high risk of DCAN were evaluated to calculate the optimum positive predictive value (PPV) and negative predictive value (NPV) in two different datasets. Furthermore, the proportion of individuals who required subsequent testing (\%Next) was compared. The confidence intervals (CIs) for the AUC, sensitivity, specificity, and predictive values were calculated using bootstrapping (1000).

\section{Results}

\section{Characteristics of the subjects}

The baseline clinical characteristics of the 455 diabetic patients have been detailed earlier ${ }^{11-14}$ and are listed in Table 1. The study cohort consisted of 208 males and 247 women (mean age, $62.80 \pm 8.61$ years). The average age of the participants in the external cohort was 58.34 years. In the study group, the mean fasting plasma glucose (FPG), postprandial blood glucose (PBG), total cholesterol (TC), and triglyceride (TG) values were $7.34,11.98,5.38$, and $1.99 \mathrm{mmol} / \mathrm{L}$, respectively. The mean HR of the study group was $75 \mathrm{bpm}$. The prevalence of HTN and MetS was $64.37 \%$ and $75.71 \%$, respectively, in the study group, and the prevalence of these two common diseases in the external cohort was $58.77 \%$ and $75.65 \%$, respectively. The prevalence of DCAN was $27.13 \%$ and $40.87 \%$ in the study group and external cohort, respectively. 


\section{Model development}

As mentioned in our previous studies, ${ }^{11-14}$ with regard to potential risk factors, age, resting HR, FPG, PBG, TG, DMD, duration of HTN, and MetS were significantly associated with DCAN according to the univariate regression analysis. In the multiple variable regression models containing all these variables, after stepwise backward elimination of the non-significant variables, the final best-fit multiple variable regression model included the following risk factors: age, FPG, PBG, TG, HR, and DMD $(P<0.05$ for all, Table 2$)$. No plausible interactions among independent variables were significantly tested (data not shown). The clinical risk model score was calculated from the regression coefficient $(\beta)$ with independent variables and continuous variables in model 1 and categorical variables in model 2 . In model 2 , the potential risk factors were categorized. Age was categorized into three groups: $\leq 50,51-65$, and $>65$ years; resting HR was categorized into four groups: $\leq 63,64-76,77-84$, and $>84$ beats/min; FPG was categorized into three groups: $<6.5 \mathrm{mmol} / \mathrm{L}, 6.5-11.5 \mathrm{mmol} / \mathrm{L}$, and $>11.5 \mathrm{mmol} / \mathrm{L} ; \mathrm{PBG}$ was categorized into two groups: $<11.5 \mathrm{mmol} / \mathrm{L}$ and $\geq 11.5 \mathrm{mmol} / \mathrm{L}$; TG was categorized into two groups: $<2.25 \mathrm{mmol} / \mathrm{L}$ and $\geq 2.25 \mathrm{mmol} / \mathrm{L}$; and $\mathrm{DM}$ duration was categorized into four groups: $<1$ year, $1-9$ years, $10-19$ years, and $\geq 20$ years.

In model 1, the total clinical risk score ranged between 8.50 and 18.16. The AUC was $0.794\left(95 \% \mathrm{CI}^{7}: 0.757-0.824\right.$, $P<0.001$, Fig. 1), which was almost identical to that of the corresponding model. A cutoff score of 12.54 was optimum (sensitivity $=68.20 \%$; specificity $=76.80 \%$; Youden index $=45.00 \%$, and $\% \mathrm{Next}=35.77 \%$, Table 3). The PPV and NPV were $54.57 \%$ and $85.53 \%$, respectively.

Table 2. MLR analysis for clinical risk model on DCAN.

\begin{tabular}{lccrcc}
\hline Variable & $\beta$ & SE & $P$ value & OR & $95.0 \%$ CI for OR \\
\hline Model 1 & & & & & \\
Age & 0.045 & 0.011 & $<0.001$ & 1.046 & $1.023-1.07$ \\
FPG & 0.112 & 0.041 & 0.006 & 1.119 & $1.033-1.211$ \\
PBG & 0.052 & 0.025 & 0.039 & 1.053 & $1.003-1.106$ \\
TG & 0.228 & 0.082 & 0.006 & 1.255 & $1.068-1.476$ \\
HR & 0.094 & 0.011 & $<0.001$ & 1.099 & $1.076-1.123$ \\
DMD & 0.347 & 0.106 & 0.001 & 1.414 & $1.15-1.74$ \\
Model 2 & & & & & \\
Age & 0.663 & 0.18 & $<0.001$ & 1.94 & $1.362-2.763$ \\
FPG & 0.702 & 0.167 & $<0.001$ & 2.018 & $1.454-2.801$ \\
PBG & 0.691 & 0.206 & 0.001 & 1.996 & $1.332-2.992$ \\
TG & 0.811 & 0.273 & 0.003 & 2.249 & $1.318-3.839$ \\
HR & 1.167 & 0.121 & $<0.001$ & 3.211 & $2.532-4.073$ \\
DMD & 0.356 & 0.107 & 0.001 & 1.427 & $1.156-1.762$ \\
\hline
\end{tabular}

Notes: For each significant variable in the MLR analysis, a clinical risk model score was calculated from the regression coefficients $(\beta)$ and independence variables.

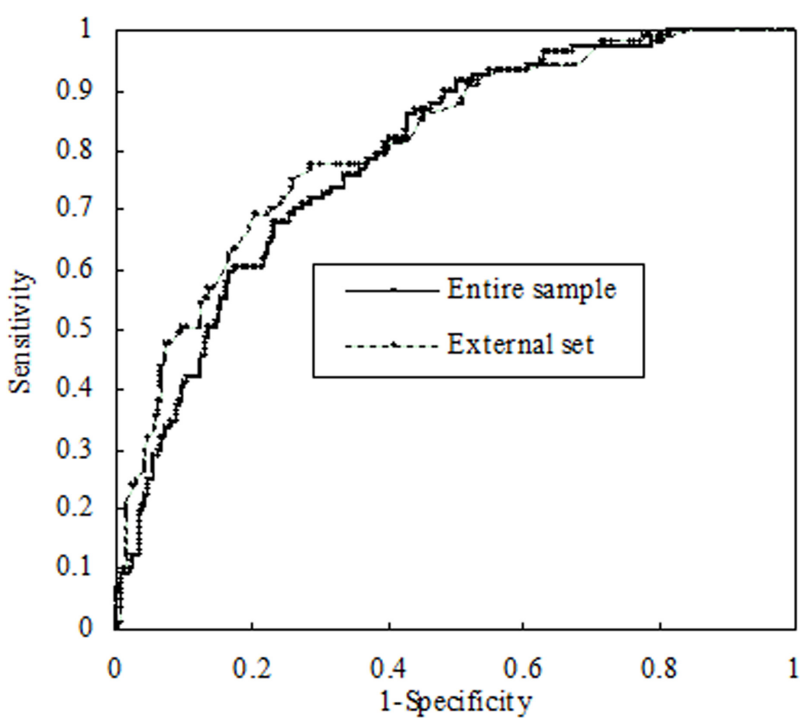

Figure 1. ROC curves showed the performance of clinical risk model 1 of DCAN in the entire sample and external dataset. The $95 \% \mathrm{CI}$ is given in parentheses. In the entire sample, AUC $=0.794,95 \%$ CI: $0.757-0.824, P<$ 0.001 and in external dataset, $\mathrm{AUC}=0.810,95 \% \mathrm{CI}$ : $0.776-0.843$, $P<0.001$.

In model 2, the AUC was 0.819 (95\% CI, 0.732-0.906, $P<0.001$, Fig. 1). The total clinical risk score ranged between 0 and 8.82. A cutoff score of 4.50 was optimum $\quad$ (sensitivity $=69.20 \% ; \quad$ specificity $=79.40 \%$; Youden index $=48.60 \%$, PPV $=57.85 \%, \mathrm{NPV}=86.32 \%$, and $\%$ Next $=34.20 \%$, Table 3).

\section{Model validation}

The clinical risk model 1 derived from the study group predicted the prevalence of DCAN in the external cohort well with an AUC of 0.810 (95\% CI: 0.776-0.843, Fig. 1). At a cutoff point of 12.54 in the external cohort, the sensitivity, specificity, and \%Next was $70.70 \%, 80.30 \%$, and $42.16 \%$, respectively. In model 2, the AUC was 0.820 (95\% CI: $0.733-0.908, P<0.001$, Fig. 2). A cutoff score of 4.50 was optimum $\quad$ (sensitivity $=68.30 \% ; \quad$ specificity $=85.20 \%$; Youden index $=53.50 \%$, PPV $=76.13 \%, \mathrm{NPV}=79.54 \%$, and $\% \mathrm{Next}=37.25 \%$, Table 3). The specificity, predictive values, and percentage of the population that required subsequent testing were similar in the two cohorts, whereas the Youden index of the external cohort tended to be higher compared with that of the study group.

\section{Performance analysis of the clinical risk models}

For clinical practice, in model 1, two cutoff points were selected to divide the study group into three risk groups: a lowrisk group (score of 8.50-11.45), medium-risk group (score of 11.46-14.70), and high-risk group (score of 14.71-18.16). 
Table 3. Performance analysis of clinical risk model for DCAN in entire sample and external set.

\begin{tabular}{|c|c|c|c|c|c|c|}
\hline \multirow[b]{2}{*}{ Model } & \multicolumn{3}{|c|}{ Model 1} & \multicolumn{3}{|c|}{ Model 2} \\
\hline & Cutoff point: 12.54 & Cutoff point: 11.45 & Cutoff point: 14.70 & Cutoff point: 4.50 & Cutoff point: 2.83 & Cutoff point: 6.00 \\
\hline \multicolumn{7}{|l|}{ Entire sample } \\
\hline Sensitivity\% & $68.20(61.85-74.55)$ & 95.3 (91.49-99.11) & $9.3(3.29-15.31)$ & $69.2(63.08-75.32)$ & $97.2(93.49-100.91)$ & $20.6(14.23-26.97)$ \\
\hline Specificity\% & $76.80(68.40-85.20)$ & $37.3(28.73-45.87)$ & 99.3 (99.03-99.57) & $79.4(70.54-88.26)$ & $28.9(20.24-37.56)$ & 98.6 (97.9-99.3) \\
\hline Yunden Index\% & $45.00(37.08-52.92)$ & $32.6(25.25-39.95)$ & $8.6(1.11-16.09)$ & $48.6(40.63-56.57)$ & $26.1(18.25-33.95)$ & $19.2(11.47-26.93)$ \\
\hline PPV\% & 54.57 (44.99-64.16) & $38.31(28.99-47.63)$ & 84.45 (74.62-94.27) & 57.85 (48.31-67.4) & $35.84(25.96-45.72)$ & 85.74 (76.69-94.8) \\
\hline NPV\% & 85.53 (76.15-94.9) & 95.1 (91.71-98.5) & $72.82(63.41-82.23)$ & $86.32(76.49-96.14)$ & 96.19 (92.31-100.07) & $75.24(66.04-84.44)$ \\
\hline$\%$ Next & $35.77(27.35-44.19)$ & $28.2(19.77-36.62)$ & $3.13(0.75-5.51)$ & $34.2(25.82-42.59)$ & 21.67 (13.21-30.13) & $6.79(5.17-8.4)$ \\
\hline \multicolumn{7}{|l|}{ External set } \\
\hline Sensitivity\% & $70.7(64.24-77.16)$ & $87.8(84.04-91.56)$ & $19.5(12.73-26.27)$ & $68.3(61.73-74.87)$ & 92.7 (88.79-96.61) & $26.8(20.43-33.17)$ \\
\hline Specificity\% & $80.3(72.06-88.54)$ & $52.5(44.11-60.89)$ & 99.9 (99.83-99.97) & $85.2(76.73-93.67)$ & $39.3(31.23-47.37)$ & $98.4(98.25-98.55)$ \\
\hline Yunden Index\% & $51(43.86-58.14)$ & $40.3(33.03-47.57)$ & $19.4(11.44-27.36)$ & $53.5(45.57-61.43)$ & $32(24.67-39.33)$ & $25.2(17.25-33.15)$ \\
\hline PPV\% & $71.27(62-80.54)$ & 56.09 (46.22-65.97) & 99.54 (99.45-99.63) & $76.13(66.84-85.42)$ & $51.35(41.58-61.12)$ & 92.05 (88.56-95.54) \\
\hline NPV\% & 79.86 (70.24-89.48) & $86.16(82.31-90.02)$ & $64.25(55.18-73.33)$ & $79.54(70.23-88.86)$ & $88.62(85.58-91.67)$ & $66.04(56.55-75.53)$ \\
\hline$\%$ Next & $42.16(33.82-50.49)$ & $36.27(27.58-44.97)$ & $7.84(5.85-9.84)$ & $37.25(29.25-45.26)$ & $25.49(17.23-33.75)$ & $10.78(9.01-12.56)$ \\
\hline
\end{tabular}

Notes: The CIs for sensitivity, specificity, and predictive values were calculated using bootstrapping (1000).

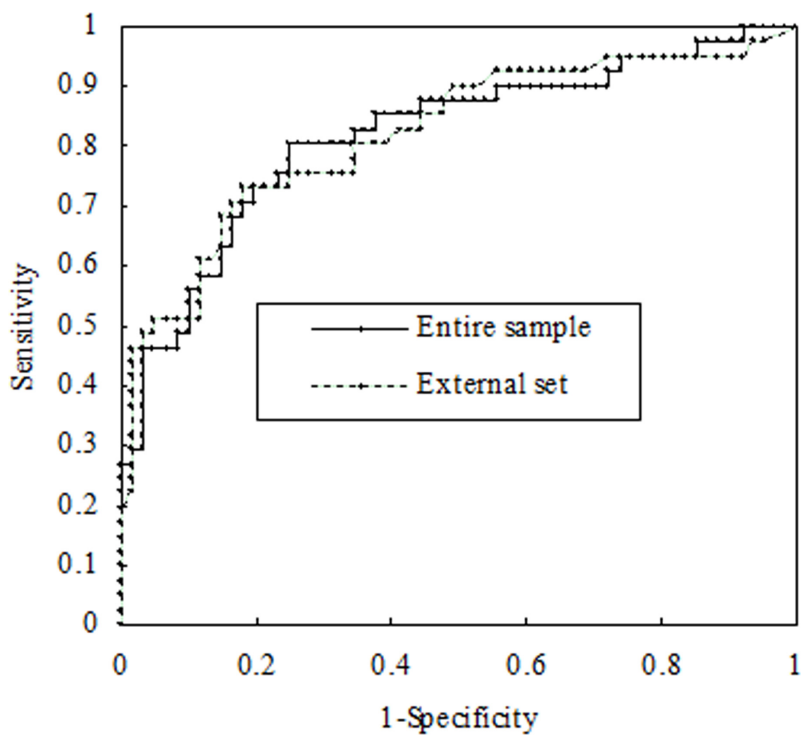

Figure 2. ROC curves showed the performance of clinical risk model 2 of DCAN in the entire sample and external dataset. The $95 \% \mathrm{CI}$ is given in parentheses. In entire sample, AUC $=0.819,95 \% \mathrm{CI}$ : 0.732-0.906, $P<$ 0.001 and in external dataset, $\mathrm{AUC}=0.820,95 \% \mathrm{CI}: 0.733-0.908$, $P<0.001$.

In the external cohort, the sensitivity at a cutoff point of 11.45 and specificity at a cutoff point of 14.70 was $87.80 \%$ and $99.90 \%$, respectively. At the cutoff point of 11.45 , the NPV was $86.16 \%$. At the cutoff point of 14.70 , the PPV was $99.54 \%$.

In model 2, the study group was also categorized into three risk groups: a low-risk group (0-2.83), medium-risk group (2.84-6.00), and high-risk group (6.01-8.82). In the external cohort, the sensitivity at a cutoff point of 2.83 and specificity at a cutoff point of 6.00 was $92.70 \%$ and $98.40 \%$, respectively. The NPV was $88.62 \%$ at the cutoff point of 2.83 , and the PPV was $92.05 \%$ at the cutoff point of 6.00 . The predictive ability of these two cutoff points for the risk of DCAN was high.

\section{Discussion}

The present study was conducted to develop a clinical risk model for predicting DCAN in a sample of diabetic patients. This is the first predictive clinical risk model for DCAN in Chinese diabetic patients. A better understanding of the predictive value of this risk model will help clinicians prevent and treat CAN.

The risk of DCAN is estimated by combining different risk factors, including age, FPG, PBG, TG, HR, and DMD, in its initial stages. In the present study, we used univariate association analysis and multiple variable regression analysis to create a best-fit multiple variable regression model, which included various risk factors (age, FPG, PBG, TG, HR, and DMD). In addition, we calculated the clinical risk score using continuous variables (model 1) and categorical variables (model 2). The ROC analysis indicated that the clinical risk model had high predictive value for DCAN (study group: AUC $=0.794$ in model 1 , and AUC $=0.819$ in model 2) The sensitivities and specificities of the clinical risk models at the optimum cutoff point ranged from 0.68 to 0.85 . The Youden index of the clinical risk models ranged from 0.450 to 0.535 . These results point to the value of these clinical risk models in predicting DCAN.

For clinical practice, in model 1, two cutoff points were selected to divide the study group into three groups: a low-risk group (scores of 8.50-11.45), medium-risk group (scores of 11.46-14.70), and high-risk group (scores of 
14.71-18.16). In model 2 , the study group sample was categorized into three groups: a low-risk group (0-2.83), medium-risk group (2.84-6.00), and high-risk group (6.018.82). Low cutoff points (11.45 in model 1 and 2.83 in model 2) had very high sensitivities, ranging from $87.8 \%$ to $97.2 \%$. In addition, the NPV (86.16-96.19\%) for DCAN was very high. Thus, someone with a score lower than the cutoff point has little probability of the disease. These findings can be used to exclude a diagnosis of DCAN in clinical practice. In contrast, high cutoff points (14.70 in model 1 and 6.00 in model 2) had very high specificities and a very high PPV for DCAN. Thus, someone with a higher score has a high probability of DCAN. As a result, high cutoff points can be used to aid the diagnosis of DCAN. The clinical risk model can be used to help clinicians diagnose the disease in clinical practice.

Over the past two decades, in the field of cardiovascular disease, early management of risk factors has contributed to a reduction in the incidence of myocardial infarction. ${ }^{20}$ The development of statistical models, such as Framingham equations, which remain the most widely used algorithm, means that it is now possible to calculate the probability of future cardiovascular events based on multiple risk factors, ${ }^{21}$ enabling targeted preventative therapy for those with the highest absolute risk. ${ }^{22}$ The identification of patients with an increased risk of type $2 \mathrm{DM}$ in the future can prevent or delay the onset of this disease. ${ }^{23}$ They can also be identified using risk-score models computed with multivariate regression models based on various diabetes-related risk factors, such as age, sex, BMI, FBG, lipid profile, and $\mathrm{BP}^{24}$ A battery of tests, which evaluate the autonomic nervous system, can be used to diagnose CAN. ${ }^{2,5}$ Despite the usefulness of these tests, they are rather cumbersome to perform, requiring trained personnel, and are seldom performed in busy clinical practices. The present study of DCAN-related factors was intended to address this issue by establishing DCAN risk models. The resulting clinical risk models are non-invasive, more convenient, and less expensive compared with conventional tests.

In a general Chinese population, our previous study ${ }^{25}$ and other studies reported that hyperglycemia and insulin resistance were significantly associated with CAN. We also found that MetS and resting HR were significantly and independently associated with $\mathrm{CAN},{ }^{26}$ and therefore they had a high value in predicting CAN in a general Chinese population. We developed a scoring system, which was based on simple parameters, such as age, waist circumference (WC), HTN and resting HR, to screen CA dysfunction. A later study showed that this system was practicable. ${ }^{18}$ To the best of our knowledge, there have been no studies of DCAN risk models, although several screening models and clinical risk models have been developed for CAN. ${ }^{10,17-19}$ In the present study, the clinical risk models for DCAN consisted of glucose profiles (FPG, PBG, and DMD), whereas our previous CAN risk models did not include these parameters. Furthermore, in our previous studies, the screening system could not identify the proportion of the entire population that required subsequent testing due to small sample sizes. ${ }^{10,18,19}$ Furthermore, there were many false-negatives with this simple screening system because various factors that affected phenotypical characteristics contributed to the outcome. In contrast, the clinical risk models for DCAN developed herein can be applied in clinical medical decision-making for Chinese diabetic patients. Diabetic patients with a high-risk score should be referred for further standard CA function tests, and the adoption of a healthier lifestyle should be advised for primary prevention.

Several limitations of the present study deserve comment. First, the clinical risk models were created based on a crosssectional study. Second, the study consisted of only Chinese diabetic patients. Thus, the findings may not be relevant to other ethnicities. Third, the sample size was moderate. Largescale studies have more power to detect disease-related risk factors for inclusion in the creation of risk models. In general, risk models based on cohort studies with a long follow-up period are more robust, and more extensive validation studies are needed.

\section{Conclusion}

The clinical risk models showed high sensitivity and specificity for the prediction of DCAN in Chinese diabetic patients, suggesting that these models may be useful in primary medical care practice for identifying Chinese diabetic patients with a high risk of DCAN.

\section{Conflict of Interest}

The authors declared that there is no conflict of interest.

\section{Acknowledgments}

We thank the grant from Shanghai Development Project of Shanghai Peak Disciplines-Integrative Medicine (20150407) and China Postdoctoral Science Foundation funded project (2017M611461).

\section{References}

1. Vinik AI, Erbas T, Casellini CM. Diabetic cardiac autonomic neuropathy, inflammation and cardiovascular disease. J Diabetes Investig 2013;4:4-18.

2. Vinik AI, Ziegler D. Diabetic cardiovascular autonomic neuropathy. Circulation 2007;115:387-97. 
3. Valensi P, Sachs RN, Harfouche B, et al. Predictive value of cardiac autonomic neuropathy in diabetic patients with or without silent myocardial ischemia. Diabetes Care 2001;24:339-43.

4. Maser RE, Mitchell BD, Vinik AI, Freeman R. The association between cardiovascular autonomic neuropathy and mortality in individuals with diabetes: A meta-analysis. Diabetes Care 2003;26:1895-1901.

5. Pop-Busui R. Cardiac autonomic neuropathy in diabetes: A clinical perspective. Diabetes Care 2010;33:434-41.

6. Pop-Busui R, Evans GW, Gerstein HC, et al. Effects of cardiac autonomic dysfunction on mortality risk in the Action to Control Cardiovascular Risk in Diabetes (ACCORD) trial. Diabetes Care 2010;33:1578-84.

7. Hajifathalian $\mathrm{K}$, Ueda $\mathrm{P}, \mathrm{Lu} \mathrm{Y}$, et al. A novel risk score to predict cardiovascular disease risk in national populations (Globorisk): A pooled analysis of prospective cohorts and health examination surveys. Lancet Diabetes Endocrinol 2015;3:339-55.

8. Blaha MJ, Cainzos-Achirica M, Greenland P, et al. Role of coronary artery calcium score of zero and other negative risk markers for cardiovascular disease: The Multi-Ethnic Study of Atherosclerosis (MESA). Circulation 2016;133:849-58.

9. Pocock SJ, McCormack V, Gueyffier F, Boutitie F, Fagard RH, Boissel JP. A score for predicting risk of death from cardiovascular disease in adults with raised blood pressure, based on individual patient data from randomised controlled trials. BMJ 2001;323:75-81.

10. Zhang L, Tang ZH, Zeng F, Li Z, Zhou L, Li Y. Clinical risk model assessment for cardiovascular autonomic dysfunction in the general Chinese population. $J$ Endocrinol Investig 2015;38:615-22.

11. Song L, Zhou L, Tang Z. An association analysis of lipid profile and diabetic cardiovascular autonomic neuropathy in a Chinese sample. Lipids Health Dis 2016;15:122.

12. Ge X, Chen H, Zhang K, Tang ZH. The analysis of blood pressure profiles and their severity in relation to diabetic cardiovascular autonomic neuropathy in the Chinese population: Preliminary analysis. J Endocrinol Investig 2016;39:891-8.

13. Fang P, Dong J, Zeng F, Tang Z. Analysis of the association between glucose profiles and beta cell function for diabetic cardiovascular autonomic neuropathy in China. J Diabetes Investig 2016;8:354-62

14. Xue Y, Lv Y, Tang Z, Dong J. Analysis of a screening system for diabetic cardiovascular autonomic neuropathy in China. Med Sci Monit 2017;23:5354-62.

15. Zeng F, Tang ZH, Li Z, Yu X, Zhou L. Normative reference of short-term heart rate variability and estimation of cardiovascular autonomic neuropathy prevalence in Chinese people. J Endocrinol Investig 2014;37:385-91.

16. Tang ZH, Wang L, Zeng F, et al. Bayesian estimation of cardiovascular autonomic neuropathy diagnostic test based on short-term heart rate variability without a gold standard. BMJ Open 2014;4:e005096.

17. Ge X, Pan SM, Zeng F, Tang ZH, Wang YW. A simple Chinese risk score model for screening cardiovascular autonomic neuropathy. PloS One 2014;9:e89623.

18. Tang ZH, Zeng F, Li Z, Zhou L. A risk score of cardiovascular autonomic dysfunction for targeted screening in the Chinese population. Int J Cardiol 2013;168:4861-2.

19. Liu J, Tang ZH, Zeng F, Li Z, Zhou L. Artificial neural network models for prediction of cardiovascular autonomic dysfunction in general Chinese population. BMC Med Inform Decis Mak 2013;13:80.

20. Gregg EW, Li Y, Wang J, et al. Changes in diabetes-related complications in the United States, 1990-2010. N Engl J Med 2014;370:1514-23.

21. Kengne AP, Patel A, Colagiuri S, et al. The Framingham and UK Prospective Diabetes Study (UKPDS) risk equations do not reliably estimate the probability of cardiovascular events in a large ethnically diverse sample of patients with diabetes: The Action in Diabetes and Vascular Disease: Preterax and Diamicron-MR Controlled Evaluation (ADVANCE) Study. Diabetologia 2010;53:821-831.

22. Simmons RK, Coleman RL, Price HC, et al. Performance of the UK prospective diabetes study risk engine and the Framingham risk equations in estimating cardiovascular disease in the EPIC-Norfolk cohort. Diabetes Care 2009; 32:708-713.

23. Investigators DT, Gerstein HC, Yusuf S, et al. Effect of rosiglitazone on the frequency of diabetes in patients with impaired glucose tolerance or impaired fasting glucose: A randomised controlled trial. Lancet 2006;368:1096-105.

24. Stern M, Williams K, Eddy D, Kahn R. Validation of prediction of diabetes by the Archimedes model and comparison with other predicting models. Diabetes Care 2008;31:1670-1.

25. Tang ZH, Zeng F, Li Z, Si Y, Zhou L. The association and predictive value analysis of metabolic syndrome on diastolic heart failure in patients at high risk for coronary artery disease. Diabetol Metab Syndr 2013;5:30.

26. Lu Y, Tang ZH, Zeng F, Li Y, Zhou L. The association and predictive value analysis of metabolic syndrome combined with resting heart rate on cardiovascular autonomic neuropathy in the general Chinese population. Diabetol Metab Syndr 2013;5:73. 